\title{
Fault Diagnosis of Roller Bearing Using Dual-Tree Complex Wavelet Transform, Rough Set and Neural Network
}

\author{
Zhixin Chen \\ Logistics School, Beijing Wuzi University, Beijing \\ 101149, PR China \\ e-mail: zxchen_05@163.com
}

\author{
Lixin Gao \\ Key Laboratory of Advanced Manufacturing \\ Technology, Beijing University of Technology, Chao \\ Yang District, Beijing, 100124, PR China \\ e-mail: lead0003@163.com
}

\begin{abstract}
In a complex field environment for modern mechanical equipment, how to identify all kinds of operational status of the rolling element bearings fastly and accurately is very important and necessary. A novel approach to automated diagnosis is introduced, which is based on feature extraction with the Dual-Tree Complex Wavelet Transform (DT-CWT), then attribute reduction with rough set theory and finally pattern recognition with Artificial Neural Network. In our experiment, 4 kinds of states on a rolling element bearing test table, including normal, pitting on inner ring, pitting on outer ring and pitting on rolling element, are adopted. The experimental results indicate that the proposed feature extraction and automated diagnosis method can extract significant feature sets from signal, and can accurately distinguish many fault pattern, and has some practical value for the on-line condition monitoring of modern industrial demands.
\end{abstract}

Keywords- Dual-Tree Complex Wavelet Transform; rough set theory; Neural Network; rolling element bearings; fault diagnosis

\section{INTRODUCTION}

The large scale and complexity of modern machines, together with the urgent needs of real-time and automatic machine fault diagnosis, have driven the transformation of fault diagnosis technology from artificial diagnosis to intelligent diagnosis. Some intelligent machine fault diagnosis systems have been developed rapidly in the past decades by successfully-applied new theories [1]. An Intelligent Diagnosis Method for Rotating Machinery Using Least Squares Mapping and a Fuzzy Neural Network is proposed in Ref. [2]. A method for mechanical faulty diagnosis based on redundant second generation wavelet package transform, neighborhood rough set and support vector machine is presented in Ref. [3]. A procedure is proposed in Ref. [4] which experimentally recognizes gears and bearings faults of a typical gearbox system using a multilayer perception neural network, and which feature vector to neural network was innovated by standard deviation of wavelet packet coefficients. Among all kinds of intelligent diagnosis methods, pattern recognition based on an Artificial Neural Network (ANN) has been widely used because of its power in self-organizing, unsupervised-learning, and nonlinear pattern classification [5].
During the signal classification, it is important to apply shift-invariant wavelet transform. Otherwise the results will be too sensitive to time locations [6-7]. A major problem of the Discrete Wavelet Transform (DWT) is lack of shift invariance. The signal information may even not be stationary in the sub-bands so that the energy distribution across the sub-bands may change [8-9]. Continuous Wavelet Transform has property of shift invariance, but it spends too long computing time. Kingsbury proposed a Dual-Tree Complex Wavelet Transform(DT-CWT) [10], which achieves approximate shift invariance and shows some applications for motion estimation and de-noising[11] .etc.. The transform yields complex wavelet coefficients via a 'dual-tree' of parallel real filter banks.

After features are extracted, ANN is used to recognize. ANN refers to structures capable of pattern recognition, classification and function approximation tasks, and has been used extensively in the civil engineering field. BP networks are the most popular ANN and have been used in this study. With the increase of target feature attribute, training time and complexity in neural network have become non-linear increase, which is bound to affect the online status monitoring and fault identification requirements. Data reduction can be realized in rough set theory by attribute reduction properties, so as to reduce training time and complexity of the network aim, and to improve the efficiency for identification of target.

Data reduction plays an important role in machine learning and pattern recognition with a high-dimensional data. In real-world applications, data usually exists with hybrid formats. Rough set theory has been proved to be a powerful tool to deal with uncertainty and has been applied to data reduction, rule extraction, data mining and granularity computation. Reduction is a minimal attribute subset of the original data which is independent and has the same discrimination power as all of the attributes in rough set framework. Obviously reduction is a feature subset selection process, where the selected feature subset not only retains the representational power, but also has minimal redundancy [12].

A fault diagnosis method for roller bearing based on nonlinear redundant lifting wavelet packet algorithm was put forward [13]. In this paper, a novel approach to automated fault diagnosis for roller bearing is introduced, which is based on feature extraction with the DT-CWT, data reduction with rough set theory and pattern recognition with 
ANN. The recognition of fault signals is important because it is very difficult to diagnose fault by the features extracted from these signals, while the approach may not need to participate diagnosing directly for worker. So it is a well studied problem.

\section{DT-CWT AND ITS APPLICATION}

The DT-CWT is a powerful tool that uses a dual tree of wavelet filters to find the real and imaginary parts of complex wavelet coefficients. Its approximate shift invariance and computational efficiency properties make it a good candidate for representing the signal features. The DTCWT of a signal is implemented using two criticallysampled DWT in parallel on the same data. For specially designed sets of filters, the wavelet associated with the upper DWT can be an approximate Hilbert transform of the wavelet associated with the lower DWT. When designed in this way, the DT-CWT is nearly shift-invariant, in contrast with the DWT. The perfect reconstruction property of the DT-CWT is verified, too.

Using multi-resolution analysis and the wavelet technique, signals can be decomposed into different frequency bands. Signals in different frequency bands can be further subject to statistical analyses to obtain feature vectors that represent signal characteristics. It is characterized by wide-frequency-range responses when processing nonstationary, transient signals with higher frequency resolution at low frequency and higher time resolution at high frequency. Compared to the FFT, it contains a great deal of non-stationary and nonlinear diagnostic information. The theoretical basis for wavelet frequency bandwidth analysis is Parseval's theorem.

Vibration signals are decomposed into independent frequency bands of different levels by using a conjugate quadrature filter. These decomposed signals agree with the law of conservation of energy, and contain a large quantity of non-stationary and nonlinear diagnostic information compared to an FFT. Therefore, the signal energy in every frequency band can be used as a feature vector to represent the operation condition of the machine and is useful for machine fault diagnosis.

DT-CWT is used to extract features from a signal in terms of its approximate shift invariance characteristics. Signal with N sampling points is decomposed into log2N levels by DT-CWT, and the 2-norm of the wavelet coefficients is computed at each of these levels. These 2norms constitute the feature vector of the signal. The 2-norm has the advantage that no back transform of the Fourier coefficients from the frequency to the time domain is necessary [7].

\section{FAULT DIAGNOSIS OF ROLLING ELEMENT BEARINGS USING DT-CWT, ROUGH SET THEORY AND NEURAL NETWORKS}

\section{A. The procedure for fault diagnosis of rolling element bearings}

All kinds of simulated fault signals are gotten from the test table in laboratory. Some of them are as training samples, the other are testing samples. Feature vectors are computed and are as condition attributes, and the initial attribute decision table is generated. Then minimal condition attributes set is obtained by attribute reduction with rough set theory, and the optimal decision table is achieved. Finally, decision table after attribute reduction is trained and tsested using neural network.

DT-CWT is used to extract features from a signal. For example, a signal with 1024 sampling points is decomposed into 10 levels, and a feature vector whose dimension is $11(11-\mathrm{D})$ is obtained after extracting features with the DTCWT.

After feature vectors are obtained they are used further to reduce dimension with rough set theory and recognize fault pattern with ANN. ANN is trained on a labelled training set and then the trained ANN is used to make classifications of an unlabeled test set. By contrastively analyzing, BP-ANN is applied and it can distinguish more effectively.

The procedure for automated diagnosis to recognize fault pattern involves the following steps:

a). All signals are normalized, which is useful for same fault pattern at different mechanical equipments.

b). Signal with $\mathrm{N}$ sampling points is decomposed into log2N levels by DT-CWT with appropriate wavelet base pairs.

c). The 2-norm of the wavelet coefficients is computed at each of these levels. A vector constituted with these 2-norms is regarded as the feature vector of the signal. This step can reduce the number of features of each signal to prevent the curse of dimensionality.

d). The feature vectors extracted are reduce dimension with rough set theory.

e). Some of the feature vectors extracted are used to train with ANN. The amount of trained feature vectors lies on actual demand.

f). The other feature vectors are used to recognize fault pattern with ANN which is trained well.

The automated diagnosis scheme based on an DT-CWT, rough set theory and ANN methods is shown in Figure 1.

\section{B. Experimental setup}

Experimental system includes the bearing test bed, HG3528A data acquisition device, notebook computer. Different pitting bearings are installed in the experimental stage, and the collected data is uploaded to the computer. The experimental platform is controlled three-phase asynchronous motor through flexible couplings, the shaft is supported by two bearings, one is a normal bearing, and the other is different pitting bearing and is installed in the B-side. Motor is constant speed of $1496 \mathrm{r} / \mathrm{min}$. The large diameter of 
bearing is $80 \mathrm{~mm}$, the small diameter is $35 \mathrm{~mm}$, number of rolling element is 8 , and the contact angle is 0 . In experiment the pitting bearing is single bit of pitting defects, which diameter size is $2 \mathrm{~mm}$, depth is $0.1 \mathrm{~mm}$. The bearing is composed of four components as the rolling element, inner ring, outer ring, and retainer. The sampling frequency is $6000 \times 2.56=15360 \mathrm{hz}$, sampling points to 8192.In our experiment, 4 kind of states on a bearing, including normal (0), pitting on inner ring (1), pitting on outer ring (2) and pitting on rolling element (3), are adopted. Four signals from each state are sampled. For these 16 signals, 11 feature vectors are obtained.

These 16 samples are regarded as the decision object. The signal energy features are regarded as conditions attributes of decision table, which are extracted by DT-CWT from each frequency band. Decision attribute is of bearing different states: normal, pitting on inner ring, pitting on outer ring and pitting on rolling element.

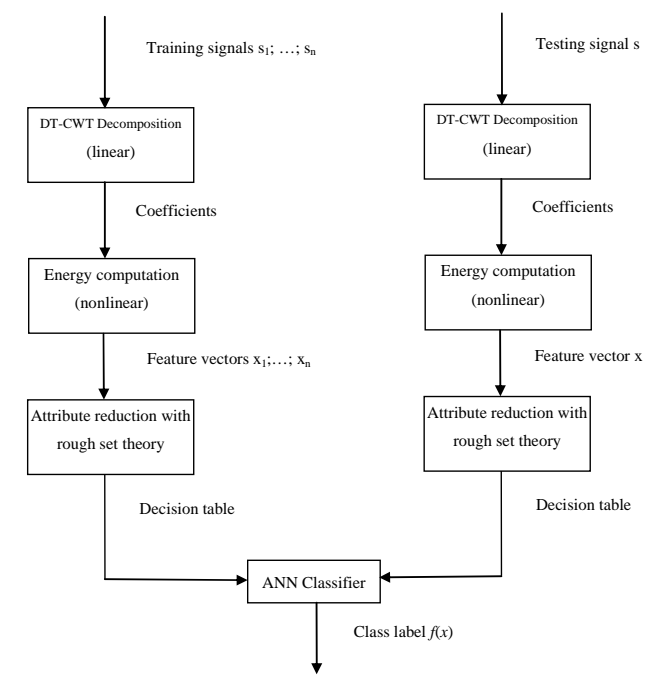

Figure 1. The principle of intelligent fault diagnosis based on DT-CWT, rough set theory and ANN

\section{Results and discussion}

After data reduction, the length of the training sample is from 11 into 6 . So the number of input layer neurons is 6 . The training samples are corresponding to the four kinds of fault type, so the number of output layer neurons is 4 . The number of hidden layer neurons is initialized as 10 .

Respectively [1000], [0100], [0010], [0001] are as output layer neurons representing normal, pitting on inner ring, pitting on outer ring and pitting on rolling element.

The previous 14 training samples are trained by BP neural network, and the neural network model is established. The other 2 testing samples are tested to verify accuracy.

The final output: before attribute reduction, a1 $=[0.8693$ 0.05710 .04680 .1060 , a2 =[ [0.0368 0.0965 0.8677 -0.0946], and the cost of time is 1'23"; after attribute reduction, a1 $=\left[\begin{array}{llll}1.0071 & -0.0675 & -0.0601 & 0.0858\end{array}\right]$, a2 $=\left[\begin{array}{lll}0.0550 & -0.1412\end{array}\right.$ $1.05750 .0819]$, and the cost of time is 53 ”.
The results is close to actual results a1 $=\left[\begin{array}{llll}1 & 0 & 0 & 0\end{array}\right], \mathrm{a} 2=\left[\begin{array}{ll}0 & 0\end{array}\right.$ 010 ], which is within the allowable range of error. It shows that the network training is successful, and attribute reduction method by rough set theory is feasible.

\section{ANALYSIS OF THE FAULT DIAGNOSIS ABILITY}

In order to describe the ability of automated diagnosis method put forward in this paper, we carried out a case which come from the practical high-speed wire rolling mill and made comparative analysis with traditional method of Fourier transform.

Case: fault diagnosis for pitting on outer ring of roller bearing in speed increasing box

In this case, 2 kinds of states on a bearing, including normal (1) and pitting on outer ring (2), are adopted. Twenty signals from each state are sampled. For these 40 signals, 11 feature vectors are obtained by DT-CWT.

The later 5 objects of 20 for each state are as the test sample, the other former 15 objects are as training samples of the neural network. Using proposed method, decision table is reduced by rough set theory, and the C1, C2, C11 are determined as optimal conditions.

The number of input layer neurons is 3. The training samples are corresponding to 2 kinds of fault type, so the number of output layer neurons is 2 . The number of hidden layer neurons is initialized as 10 . Respectively [1 0], [0 1] are as output layer neurons representing normal and pitting on outer ring. The previous 15 training samples are trained by BP neural network, and the neural network model is established. The other 5 testing samples are tested to verify accuracy.

The results show that the recognized accuracy reaches $100 \%$.

Artificially, through Fourier Transform analysis of the original vibration signals, the method shows that the rotary frequency Shaft I in speed increasing box in some factory was $39.06 \mathrm{~Hz}$. Figure 2(a, b) shows the original signal and the spectrum after Fourier Transform. Figure 2(c, d) shows the spectrum after DT-CWT transform reconstruction and decomposition to the data at level 6. It can be seen that the SNR of DT-CWT is higher in the spectrum analysis and multiple frequency emerge to account for pitting on outer ring of roller bearing. The device disintegrated later; the pitting on outer ring is shown in Figure 3.

Figure 4 shows the signal of under normal condition of one rolling mill and can be seen that fault is not obvious.
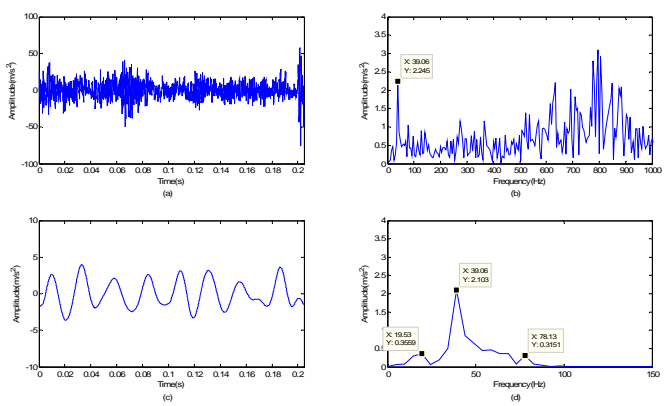
Figure 2. Comparison analysis between Fourier Transform and DT-CWT. (a) Time domain waveform of the original signals; (b) Spectrum of the original signals after Fourier Transform; (c) Time domain waveform of approximation signal reconstruction after decomposition of DT-CWT at level 6; (d) Spectrum analysis of (c)

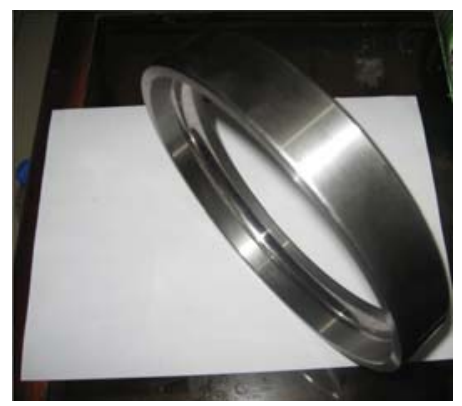

Figure 3. The real case of pitting on outer ring of angle bearing in shaft I in speed increasing box
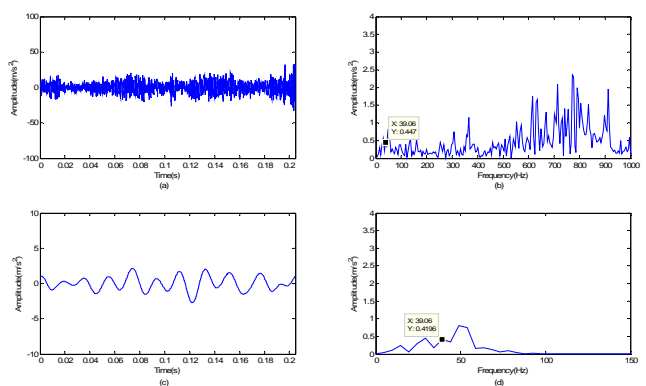

Figure 4. Comparison analysis between Fourier Transform and DT-CWT under normal condition. (a) Time domain waveform of the original signals;

(b) Spectrum of the original signals after Fourier Transform; (c) Time

domain waveform of approximation signal reconstruction after

decomposition of DT-CWT at level 6; (d) Spectrum analysis of (c)

\section{CONCLUSION}

The approximate shift invariance and computational efficiency properties make DT-CWT a good candidate for representing the signal features.

Integrating different diagnosis technologies has become popular in intelligent diagnosis research. Firstly, status features are extracted from the original signal with the DTCWT, and then redundant or unimportant attribute features are removed with rough set theory. Lastly, a pattern is recognized for fault diagnosis with ANN based on BP. The method can improve the neural network classifier in learning accuracy, performing efficiency. So it has some practical value for the on-line condition monitoring of modern industrial demands.

The experimental results indicate that the proposed approach, which is based on feature extraction with the DTCWT, attribute reduction with rough set theory, and pattern recognition with ANN, can extract significant feature sets from signal, and can accurately distinguish much fault pattern. So it can be efficient and useful in automated diagnosis to machinery fault.

\section{ACKNOWLEDGMENT}

This work was financially supported by the Organization Department of Beijing Talents Project (Project No.2012D005009000002), and the Funds for Distinguished Young Scientists of Beijing Wuzi University(Project No.2012XJQN014).

\section{REFERENCES}

[1] Lixin Gao, Zhiqiang Ren, Wenliang Tang, Huaqing Wang, and Peng Chen. Intelligent Gearbox Diagnosis Methods Based on SVM, Wavelet Lifting and RBR. Sensors 2010, 10, 4602-4621

[2] Ke Li, Peng Chen and Shiming Wang. An Intelligent Diagnosis Method for Rotating Machinery Using Least Squares Mapping and a Fuzzy Neural Network. Sensors 2012, 12, 5919-5939

[3] Ning Li, Rui Zhou, Qinghua Hu, Xiaohang Liu. Mechanical fault diagnosis based on redundant second generation wavelet packet transform, neighborhood rough set and support vector machine. Mechanical Systems and Signal Processing 28(2012)608-621

[4] J. Rafiee, F. Arvani, A. Harifi, M.H.Sadeghi. Intelligent condition monitoring of a gearbox using artificial neural network. Mechanical Systems and Signal Processing 21 (2007) 1746-1754

[5] Jack, L.B.; Nandi, A.K.; McCormick, A.C. Diagnosis of roller bearing faults using radial basis function net works. Appl. Signal Process. 6(1999) 25-32

[6] X.Xu, M.Kezunovic. Automated Feature Extraction From Power System Transients Using Wavelet Transform. IEEE, 4(2002) 1994 1998

[7] Julia Neumann, Gabriele Steidl. Dual-tree complex wavelet transform in the frequency domain and an application to signal classification. International Journal of Wavelets, Multiresolution and Information Processing. 2005, 3(1):43-65

[8] E. P. Simoncelli, W. T. Freeman, E. H. Adelson, and D. J. Heeger. Shiftable multiscale transforms. IEEE Transactions on Information Theory, MIT Media Laboratory Vision and Modeling Technical Report, March 1992. No.161. 38(2):587-607.

[9] N. G. Kingsbury. Complex wavelets for shift invariant analysis and filtering of signals. Journal of Applied and Computational Harmonic Analysis, May 2001, 10(3):234-253.

[10] G. Kingsbury. The dual-tree complex wavelet transform: a new technique for shift invariance and directional filters. In: Proceedings of 8th IEEE Digital Signal Processing Workshop, Bryce Canyon, Utah, USA, 1998:86-89.

[11] N. G. Kingsbury, J. F. A. Magarey. Wavelet transforms in image processing. In Proc. First European Conference on Signal Analysis and Prediction, ICT Press, 1997:23-34.

[12] Qinghua Hu, Daren Yu, Zongxia Xie. Information-preserving hybrid data reduction based on fuzzy-rough techniques. Pattern Recognition Letters 27 (2006) 414-423

[13] Lixin Gao, Zijing Yang, Ligang Cai, Huaqing Wang and Peng Chen. Roller Bearing Fault Diagnosis Based on Nonlinear Redundant Lifting Wavelet Packet Analysis. Sensors 2011, 11, 260-277 